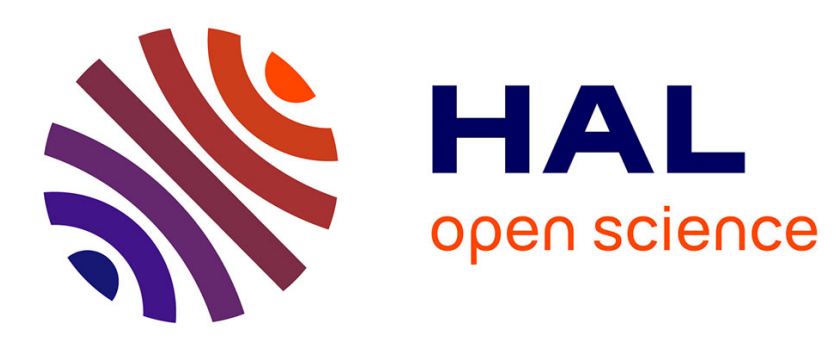

\title{
Oxidation-Responsive Emulsions Stabilized by Cleavable Metallo-Supramolecular Cross-Linked Microgels
}

\author{
Julien Es Sayed, Christophe Meyer, Nicolas Sanson, Patrick Perrin
}

\section{To cite this version:}

Julien Es Sayed, Christophe Meyer, Nicolas Sanson, Patrick Perrin. Oxidation-Responsive Emulsions Stabilized by Cleavable Metallo-Supramolecular Cross-Linked Microgels. ACS Macro Letters, 2020, pp.1040-1045. 10.1021/acsmacrolett.0c00389 . hal-02896994

\section{HAL Id: hal-02896994 \\ https://hal.science/hal-02896994}

Submitted on 24 Nov 2020

HAL is a multi-disciplinary open access archive for the deposit and dissemination of scientific research documents, whether they are published or not. The documents may come from teaching and research institutions in France or abroad, or from public or private research centers.
L'archive ouverte pluridisciplinaire HAL, est destinée au dépôt et à la diffusion de documents scientifiques de niveau recherche, publiés ou non, émanant des établissements d'enseignement et de recherche français ou étrangers, des laboratoires publics ou privés. 
Oxidation-Responsive Emulsions Stabilized by Cleavable Metallo-Supramolecular Crosslinked Microgels

Julien Es Sayed ${ }^{\dagger}$, Christophe Meyer ${ }^{\ddagger}$, Nicolas Sanson ${ }^{\dagger *}$, Patrick Perrin $^{\dagger *}$

${ }^{\dagger}$ Soft Matter Sciences and Engineering, ESPCI, PSL University, Sorbonne Université, CNRS, 10 rue Vauquelin, 75231, Paris Cedex 05, France.

${ }^{\ddagger}$ Molecular, Macromolecular Chemistry and Materials, ESPCI, PSL University, CNRS, 10 rue Vauquelin, 75231, Paris Cedex 05, France.

KEYWORDS: responsive emulsion, supramolecular chemistry, microgel, oxidation, terpyridine complex

\begin{abstract}
An original route to develop an advanced class of microgels emulsifiers containing stimulable metallo-supramolecular instead of frozen covalent crosslinks is reported. The poly $(N-$ isopropylmethacrylamide) (PNiPMAM) chains of the microgel are connected by iron(II)bis(terpyridine) coordination supramolecular complexes that can be cleaved on demand leading to unique properties both at interfaces and in volume. The microgels synthesis is undemanding and the characterization of their supramolecular structure can be precisely achieved by standard methods. Singularly, interfaces of an oil-in-water emulsion stabilized by the supramolecular particles can be triggered at molecular scale by oxidation of $\mathrm{Fe}(\mathrm{II})$ to $\mathrm{Fe}(\mathrm{III})$, leading to emulsion breaking. In bulk, we show that a microgel dispersion can indeed be transformed into a polymer solution upon oxidation. Our study paves the road to the discovery of unusual microgel properties as our proof-of-concept can be extended to different supramolecular chemistry and architecture.
\end{abstract}


Microgel engineering has become a tremendous field of research due to the highly tunable structure of these crosslinked soft and thus deformable particles. ${ }^{1-6}$ For more than a decade, microgels have been extensively used as emulsion particle stabilizers. ${ }^{7-9}$ Similarly to hard particles, microgels provide a remarkable kinetic stability to emulsions due to their irreversible adsorption at interfaces leading to the well-known limited coalescence behavior peculiar to the Ramsden-Pickering emulsions. ${ }^{10,11}$ However, in contrast to hard particles stabilized emulsions, stabilization mechanisms operating for adsorbed microgels are complex as microgels are shown to flatten at the interface to adopt the so-called fried-egg conformation. $^{12-16}$ Nevertheless, that a higher deformability of the microgels improves interfacial elasticity and hence emulsion stability seems to emerge as a general consensus among the different reports. ${ }^{17-19}$ In line with it, any trigger that modifies the deformability of adsorbed microgels has been used to affect emulsion stability. Thus, responsive destabilization of emulsions formulated with thermo- and $\mathrm{pH}$-sensitive microgels was reported using stimuli such as temperature, $\mathrm{pH}$ or ionic strength. ${ }^{20-25}$

In all studies, monitoring stability and triggering emulsion breaking were investigated within the framework of chemical microgels. In such cases, as polymer chains are covalently linked, changing deformation by modulating in-situ the degree of crosslinking of the microgels upon stimulation is not an alternative, putting aside particle degradation that usually requires rather harsh and poorly controlled chemistry. Nevertheless, it was suggested that $\mathrm{pH}$ control provides the opportunity to vary the number of $\mathrm{H}$-bonds and thus the number of effective crosslinks in covalent networks containing carboxylic acid units. ${ }^{17}$ Then, it seems interesting to add to the polymer chains network weak bonds that can be broken by stimulating the soft particles. Probing the potential of supramolecular chemistry embedded into microgels to trigger interfacial properties in general appears truly challenging. ${ }^{26-28}$ In particular, it is likely to provide systems such as emulsions with new properties.

Herein, we report on an elegant strategy to trigger emulsion destabilization by designing microgels with metallo-supramolecular chemistry-based crosslinkers. We have chosen to report on microgels containing only supramolecular crosslinks as our goal is to explore conditions going beyond the simple deformation/stability duality detailed above. As a matter of fact, emulsion stability is probed under uncommon conditions since particles are broken up into free polymer chains upon stimulation. It also gives the opportunity to investigate in detail the breaking of microgels dispersed in bulk into fragmented polymer chains as reported in the literature. ${ }^{29,30}$ Practically, we have designed microgels using terpyridine-based metal-ligand physical crosslinkers only as it theoretically affords to choose from a broad range of bond 
dynamics depending on the nature and oxidation degree of the metal cation. ${ }^{31-34}$ We show that the network structure of the synthesized metallo-supramolecular microgels can be broken on demand by oxidation leading to the emulsion destabilization (Scheme 1).

Scheme 1. General scheme for oxidative destabilization of metallo-supramolecular crosslinked microgels stabilized oil-in-water emulsion. The oxidation of iron (II) triggers the cleavage of the supramolecular crosslinks and then the destructuration of microgels into individual polymer chains leading to emulsion destabilization.

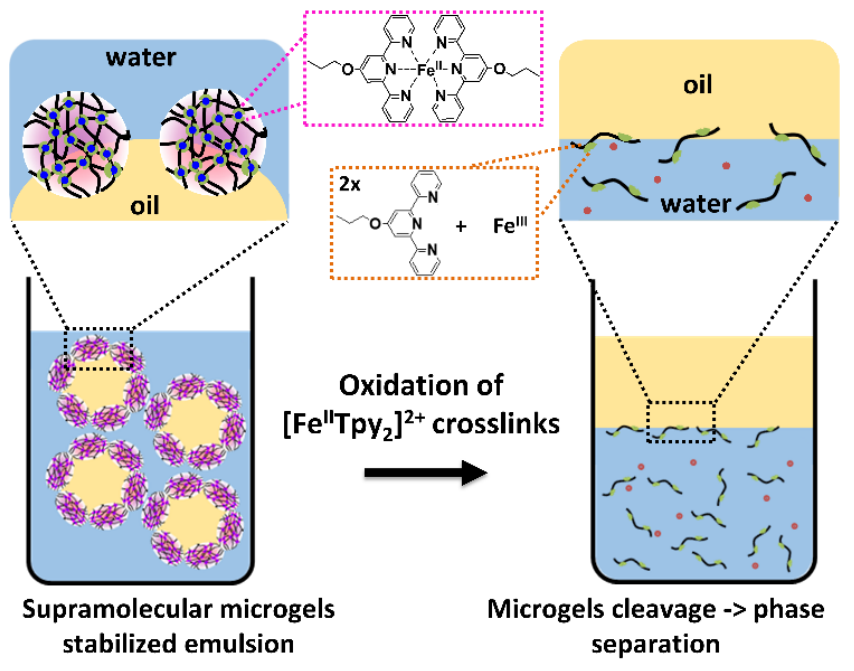

The metallo-supramolecular microgels (supra-MGs) were obtained by a two-step surfactantfree dispersion copolymerization of $N$-isopropylmethacrylamide, NiPMAM, in the presence of a terpyridine-based semi-crosslinker (TpyAm) and without any added chemical crosslinker, contrary to most classical microgels synthesis. The synthetic procedure and the characterization for both TpyAm and supra-MGs are described in Figure 1a and Figures S1S3 in the Supporting Information. In a first step, poly(NiPMAM-co-TpyAm) growing chains collapse to form water-insoluble nuclei during polymerization at $70{ }^{\circ} \mathrm{C}$ leading to monodisperse microgel-like particles without permanent links (Supporting Information, Figure S4a). ${ }^{35,36}$ The uncrosslinked nature of the growing pre-microgels is evidenced by taking a turbid sample from the reactive medium after polymerization for 4 hours and cooling 
it down below the Lower Critical Solution Temperature (LCST) of PNiPMAM. ${ }^{37,38}$ The medium becomes totally transparent to the naked eyes meaning that the formed aggregates dissociate in polymer chains (Supporting Information, Figure S4b). Moreover, DLS measurements do not show any scattering signal, which indicates the absence of microgel particles. In the second step, $\mathrm{FeCl}_{2} \bullet 4 \mathrm{H}_{2} \mathrm{O}$ was added to the reaction medium after 4 hours polymerization at $70{ }^{\circ} \mathrm{C}$ to allow for crosslinking and thus formation of the supramolecular microgels (Figure 1b and 1c). Indeed, tridentate terpyridine ligands present in the premicrogels immediately chelate iron(II) to form coordination complexes $\left[\mathrm{Fe}^{\mathrm{II}} \mathrm{Tpy}_{2}\right]^{2+}, 2 \mathrm{Cl}^{-}$as evidenced by the typical purple color of the microgel solution (Figure 1; Supporting Information, Figure S4c) ${ }^{39,40}$ It is worth noting that this two-step process generates welldefined microgels with a doubly charged crosslinker exhibiting a polyelectrolyte behavior, which partly prevents their temperature-induced deswelling above the LCST of NiPMAM polymer chains. However, increasing the ionic strength of the medium gradually screens the electrostatic interactions leading to a complete recovery of both the volume deswelling ratio and the phase transition enthalpy of the chemically crosslinked PNiPMAM microgels (Supporting Information, Figure S5). ${ }^{37}$

Then, a key feature of our approach in the design of the supra-MGs comes from the possibility to precisely quantify the effective amount of introduced crosslinkers in the network, a characterization that is most often not attainable. Indeed, the $\left[\mathrm{Fe}^{\mathrm{II}} \mathrm{Tpy}_{2}\right]^{2+}$ complex exhibits a well-pronounced absorption band at $556 \mathrm{~nm}$ due to the metal to ligand charge transfer (MLCT) identified using UV/Vis spectroscopy (Figure 2a). ${ }^{39,40}$ In the present case, the effective crosslinking ratio of supra-MGs is $1.1 \mathrm{~mol} \%$ relatively to NiPMAM repeat units (Supporting Information, Figure S6). We thus studied the effect of an external stimulus on the cleavage of the supramolecular complex and on the structure of the microgels. The responsive cleavage of metal-bis(terpyridine) based complexes can be induced either by the addition of a strong competitive ligand or by increasing $\mathrm{pH}$ to precipitate the metal of the complex. ${ }^{41-43} \mathrm{We}$ have chosen to carry out an oxidation reaction to break the metal-ligand complex. More precisely, hydrogen peroxide $\left(\mathrm{H}_{2} \mathrm{O}_{2}\right)$ and potassium persulfate (KPS) were used to oxidize into iron (III) the iron(II) embedded between the terpyridine units. ${ }^{44,45}$ The pictures presented on Figure 2b clearly show that 12 hours after addition of either $\mathrm{H}_{2} \mathrm{O}_{2}$ or KPS to the microgel dispersion, the pink color has totally vanished to give a non-turbid colorless solution. The disappearance of the $\left[\mathrm{Fe}^{\mathrm{II}} \mathrm{Tpy}_{2}\right]^{2+}$ complex is also evidenced for both oxidizer molecules by the loss of the absorption band at $556 \mathrm{~nm}$, characteristic of the Fe(II) complex, in the UV/Vis spectrum (Figure 2a). According to the literature, the formed iron(III) bis-terpyridine 
complexes are not kinetically stable and exhibit a very high lability. ${ }^{46-48}$ Considering now the consequences at the microgels scale, a rapid loss of the scattered intensity was observed five minutes after the addition of $\mathrm{H}_{2} \mathrm{O}_{2}$ (Figure 2c; Supporting Information, Figure S7 for KPS). The loss of scattering intensity indeed reflects the dissociation of the microgels into linear chains as the latter do not scatter light unlike colloidal microgels, at the same polymer concentration. Similarly, SEM observations reveal a grey-level background without visible microgels (Figure 2d). As a conclusion, the results unambiguously show that the network structure of the supra-MGs is broken by oxidation releasing free polymer PNiPMAM chains (Supporting Information, Figure S8). In other words, the microgel dispersion noteworthy changes into polymer solution under the action of the oxidation stimulus. The key feature indeed resides in the use of supramolecular chemistry that enables to open the $\left[\mathrm{Fe}^{\mathrm{II}} \mathrm{Tpy}_{2}\right]^{2+}$ complex to break the microgel network, in line with our strategy.

The present supra-MGs microgels, fully destructible in aqueous solution by oxidation, also exhibit excellent emulsifying properties. In fact, they are able to stabilize creamed dodecanein-water dispersions at very low concentrations. Evidencing their high kinetic stability, we observed that the creamed emulsions were stable for months.

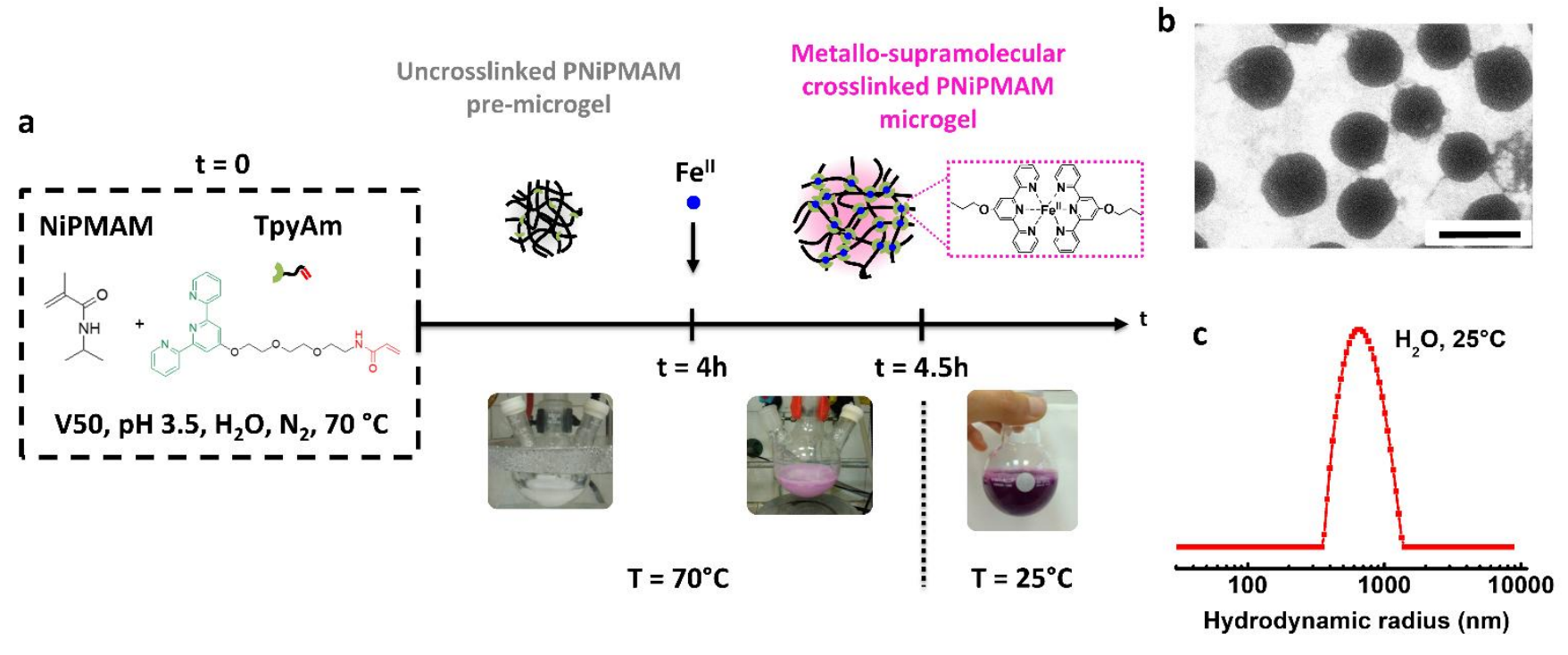

Figure 1. (a) Reaction pathway to the synthesis of metallo-supramolecular crosslinked microgels, supra-MGs. (b) SEM picture of synthesized supra-MGs (scale bar is $1 \mu \mathrm{m}$ ) (c) Intensity averaged size distribution of supra-MGs hydrodynamic radius at $25{ }^{\circ} \mathrm{C}$ in water determined by Dynamic Light Scattering (DLS). 
The supra-MGs thus seem to present all the characteristics of the Ramsden-Pickering emulsions stabilizers (Supporting Information, Figure S9). ${ }^{10,11}$ This was actually confirmed by evidencing the so-called limited coalescence regime leading to emulsions with narrow droplet size distribution. Under given stirring and concentration conditions, all microgels adsorb at the oil-water interface (Supporting Information, Figure S9a). Consequently, there is no microgel reservoir in the continuous aqueous phase of the emulsions. Macroscopically, this is indeed the case as the aqueous sub-phase in neither colored nor turbid to the naked eyes (Supporting Information, Figure S9b). Moreover, UV/Vis spectroscopy confirms the absence of microgel in water since the spectrum does not exhibit the absorption band at $556 \mathrm{~nm}$ (Supporting Information, Figure S9c). We have then investigated the effect of an oxidizing agent like $\mathrm{H}_{2} \mathrm{O}_{2}$ or KPS on emulsion stability. An aliquot of a concentrated $\mathrm{H}_{2} \mathrm{O}_{2}$ or KPS solution was added to the water sub-natant phase of the creamed emulsion to target a final $\mathrm{H}_{2} \mathrm{O}_{2}$ (resp. KPS) concentration of $10^{-2} \mathrm{M}$ (resp. $10^{-3} \mathrm{M}$ ). At the macroscopic scale, the very beginning of emulsion destabilization and a slight loss of pink color are observed $1.5 \mathrm{~h}$ after $\mathrm{H}_{2} \mathrm{O}_{2}$ /KPS addition without stirring the emulsion sample (Figure 3a; Supporting Information, Movie S1). After $3.5 \mathrm{~h}$, the sample has completely lost its pink color and the two liquids, oil and water, are almost completely phase separated even if a few droplets have not yet coalesced. The phase separation is fully achieved about $6 \mathrm{~h}$ after addition of the oxidizer. It is worth noticing that the

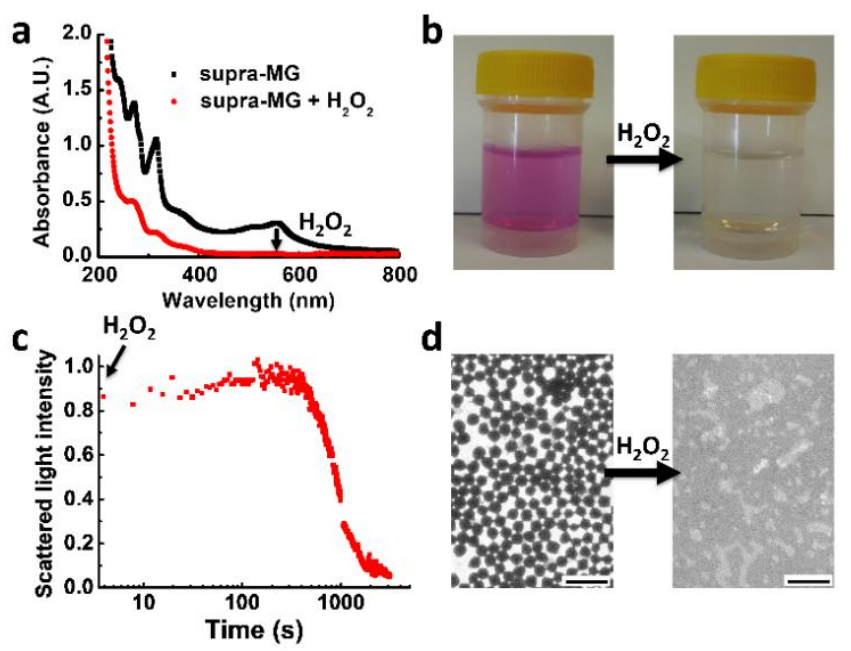

Figure 2. (a) UV/Vis spectra before and after supra-MGs oxidation by $\mathrm{H}_{2} \mathrm{O}_{2}$ at $\mathrm{C}_{M G}=0.25$ $\mathrm{mg} / \mathrm{mL}$. (b) Aspect of supra-MGs samples at a concentration of $\mathrm{C}_{\mathrm{MG}}=0.25 \mathrm{mg} / \mathrm{mL}$ before and after oxidation with $\mathrm{H}_{2} \mathrm{O}_{2}$ at $10^{-2} \mathrm{M}$. (c) Evolution of the light scattered intensity of the 
supra-MG relative to the $\mathrm{t}_{0}$ when adding $\mathrm{H}_{2} \mathrm{O}_{2}$ at $\mathrm{C}_{\mathrm{MG}}=0.25 \mathrm{mg} / \mathrm{mL}$ at $25{ }^{\circ} \mathrm{C}$. (d) $\mathrm{SEM}$ picture of supra-MGs before and after oxidation. Scale bar is $2.5 \mu \mathrm{m}$.

destabilized emulsion could not be re-emulsified using the Ultra-Turrax device even if the resulting free PNiPMAM chains are still surface active (Supporting Information, Figure S10). The use of confocal laser scanning microscopy (CLSM) enables to get an insight into the mechanism by which emulsions undergo destabilization (Figure 3b; Supporting Information, Movie S2). First, nile-blue chemically-stained fluorescent supra-MGs clearly appear to be adsorbed onto the surface of the dodecane droplets deposited on the glass slide. Upon addition of $\mathrm{H}_{2} \mathrm{O}_{2}$, a rather fast coalescence of the droplets is observed after $1 \mathrm{~min}$, concomitant with the loss of the observed round shape at the interface. Then, after $4 \mathrm{~min}$, no microgel is discernible and fluorescence at the interface is lost. Importantly, we have checked that the addition of $\mathrm{H}_{2} \mathrm{O}_{2}$ in this concentration range does not alter nile blue fluorescence thus making our observations reliable (Supporting Information, Figure S11). As a matter of fact, the loss of apparent fluorescence is rather due to the dilution of the whole fluorescence in the continuous phase, as a direct consequence of the destructuration of the microgels into linear PNiPMAM fluorescent chains. Consequently, oxidation of iron(II) into iron(III) triggers the breaking of the microgels network into individual polymer chains and the destabilization of the direct emulsion.

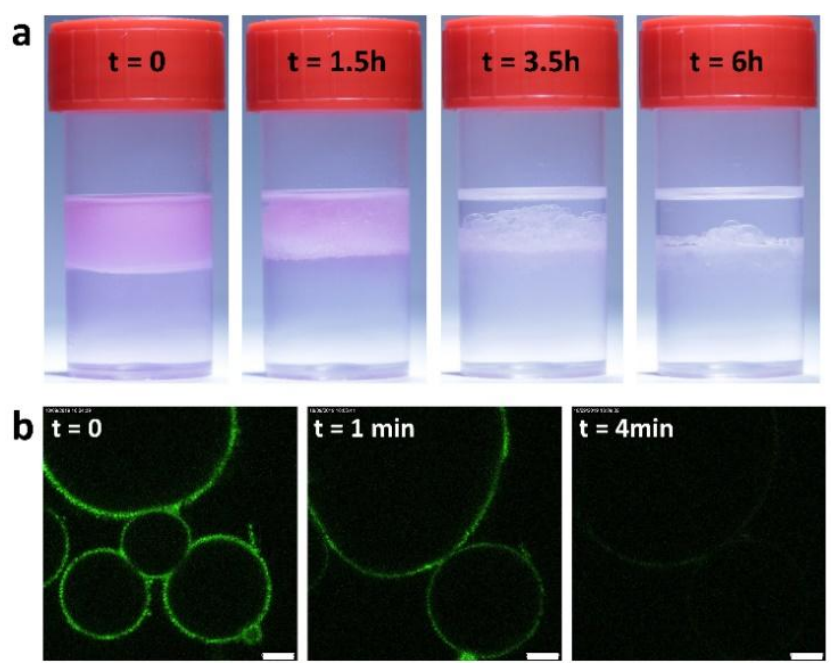

Figure 3. Following-up of the destabilization of the emulsion of dodecane in water stabilized by $\mathrm{C}_{\mathrm{MG}}=0.25 \mathrm{mg} / \mathrm{mL}$ by oxidation of supra-MGs adsorbed at oil-water interface at (a) macro-scale and (b) micro-scale by CLSM (scale bar is $10 \mu \mathrm{m}$ ). 
As a conclusion, we disclosed an original and straightforward way to design metallosupramolecular microgels by taking advantage of the insolubility of P(NiPMAM-co-TpyAm) growing chains to prepare uncrosslinked pre-microgels colloidally stabilized by the charged initiator. The supramolecular crosslinks were then generated by introducing iron(II) cations, which instantaneously undergo chelation by two terpyridine units attached to the network of the pre-microgels. Noteworthy, the accurate quantification of the crosslinking degree by means of UV-Visible spectroscopy is a strength of our microgel design. We anticipate that the supramolecular crosslinked microgels are certainly excellent tools to move forwards with the knowledge of the ongoing debate of the mechanisms involved into the microgels stabilized emulsions. This arises from the possibility to "switch on/off" the crosslinks by a thorough control of the supramolecular complexes opening leading to an in-situ precise adjustment of the microgel deformability. Beyond that, we also demonstrate that oxidation of iron(II) into iron(III) successfully triggers complete destructuration of the supramolecular microgels into linear polymer chains at oil-water interfaces. This enables to develop responsive RamsdenPickering emulsions where the rarely used oxidation stimulus triggers total phase separation. We hope that our work will stimulate research in the synthesis of microgels based on supramolecular chemistry as novel properties could be discovered both in bulk and at interfaces.

\section{ASSOCIATED CONTENT}

\section{Supporting Information.}

This material is available free of charge via the Internet at http://pubs.acs.org.

Experimental section detailing the general procedure and synthesis of the supra-mGs, polyelectrolyte behavior of the supra-MGs, Ramsden-Pickering behavior of emulsions stabilized by supra-MGs, evidence and following-up of supra-MGs cleavage upon oxidation.

\section{AUTHOR INFORMATION}

\section{Corresponding Author}

*E-mail: patrick.perrin@espci.fr

*E-mail: nicolas.sanson@espci.fr

\section{Author Contributions}


The manuscript was written through contributions of all authors. All authors have given approval to the final version of the manuscript.

\section{Notes}

The authors declare no competing financial interest.

\section{ACKNOWLEDGMENT}

The authors gratefully acknowledge the financial support of CNRS, ESPCI, and the PhD school of Sorbonne Université (ED 397, Sorbonne Université) for the PhD fellowship funding of J. Es Sayed. The authors thank Mauricio Balarezo, Sandrine Laquerbe and Thomas Pons for their helpful support to this work. The authors thank the microscopy platform of Institut Pierre Gilles de Gennes for technical advice on CLSM. The authors thank the Région Ile-deFrance for funding of SEM utilities.

\section{REFERENCES}

(1) Saunders, B. R.; Vincent, B. Microgel Particles as Model Colloids: Theory, Properties and Applications. Adv. Colloid Interface Sci. 1999, 80, 1-25.

(2) Nayak, S.; Lyon, L. A. Soft Nanotechnology with Soft Nanoparticles. Angew. Chem. Int. Ed. 2005, 44, 7686-7708.

(3) Plamper, F. A.; Richtering, W. Functional Microgels and Microgel Systems. Acc. Chem. Res. 2017, 50, 131-140.

(4) Karg, M.; Pich, A.; Hellweg, T.; Hoare, T.; Lyon, L. A.; Crassous, J. J.; Suzuki, D.; Gumerov, R. A.; Schneider, S.; Potemkin, Igor. I.; Richtering, W. Nanogels and Microgels: From Model Colloids to Applications, Recent Developments, and Future Trends. Langmuir 2019, 35, 6231-6255.

(5) Suzuki, D.; Horigome, K.; Kureha, T.; Matsui, S.; Watanabe, T. Polymeric Hydrogel Microspheres: Design, Synthesis, Characterization, Assembly and Applications. Polymer Journal 2017, 49, 695-702.

(6) Oberdisse, J.; Hellweg, T. Recent Advances in Stimuli-Responsive Core-Shell Microgel Particles: Synthesis, Characterisation, and Applications. Colloid Polym Sci 2020, DOI 10.1007/s00396-020-04629-0.

(7) Kwok, M.; Sun, G.; Ngai, T. Microgel Particles at Interfaces: Phenomena, Principles, and Opportunities in Food Sciences. Langmuir 2019, 35, 4205-4217. 
(8) Schmitt, V.; Destribats, M.; Backov, R. Colloidal Particles as Liquid Dispersion Stabilizer: Pickering Emulsions and Materials Thereof. C. R. Phys. 2014, 15, 761-774.

(9) Richtering, W. Responsive Emulsions Stabilized by Stimuli-Sensitive Microgels: Emulsions with Special Non-Pickering Properties. Langmuir 2012, 28, 17218-17229.

(10) Ramsden, W. Separation of Solids in the Surface-Layers of Solutions and "Suspensions" (Observations on Surface-Membranes, Bubbles, Emulsions, and Mechanical Coagulation). - Preliminary Account. Proc. R. Soc. London 1903, 72, 156-164.

(11) Pickering, S. U. Pickering : Emulsions. In Emulsions; 1907.

(12) Brugger, B.; Rütten, S.; Phan, K.-H.; Möller, M.; Richtering, W. The Colloidal Suprastructure of Smart Microgels at Oil-Water Interfaces. Angew. Chem. Int. Ed. 2009, 48, 3978-3981.

(13) Geisel, K.; Isa, L.; Richtering, W. Unraveling the 3D Localization and Deformation of Responsive Microgels at Oil/Water Interfaces: A Step Forward in Understanding Soft Emulsion Stabilizers. Langmuir 2012, 28, 15770-15776.

(14) Pinaud, F.; Geisel, K.; Massé, P.; Catargi, B.; Isa, L.; Richtering, W.; Ravaine, V.; Schmitt, V. Adsorption of Microgels at an Oil-Water Interface: Correlation between Packing and 2D Elasticity. Soft Matter 2014, 10, 6963-6974.

(15) Destribats, M.; Lapeyre, V.; Wolfs, M.; Sellier, E.; Leal-Calderon, F.; Ravaine, V.; Schmitt, V. Soft Microgels as Pickering Emulsion Stabilisers: Role of Particle Deformability. Soft Matter 2011, 7, 7689-7698.

(16) Harrer, J.; Rey, M.; Ciarella, S.; Löwen, H.; Janssen, L. M. C.; Vogel, N. StimuliResponsive Behavior of Poly(N-Isopropylacrylamide) Microgels under Interfacial Confinement. Langmuir 2019, 35, 10512-10521.

(17) Massé, P.; Sellier, E.; Schmitt, V.; Ravaine, V. Impact of Electrostatics on the Adsorption of Microgels at the Interface of Pickering Emulsions. Langmuir 2014, 30, 1474514756.

(18) Destribats, M.; Eyharts, M.; Lapeyre, V.; Sellier, E.; Varga, I.; Ravaine, V.; Schmitt, V. Impact of PNIPAM Microgel Size on Its Ability To Stabilize Pickering Emulsions. Langmuir 2014, 30, 1768-1777.

(19) Li, Z.; Richtering, W.; Ngai, T. Poly(N-Isopropylacrylamide) Microgels at the OilWater Interface: Temperature Effect. Soft Matter 2014, 10, 6182-6191.

(20) Ngai, T.; Behrens, S. H.; Auweter, H. Novel Emulsions Stabilized by pH and Temperature Sensitive Microgels. Chem. Comm. 2005, 3, 331-333. 
(21) Ngai, T.; Auweter, H.; Behrens, S. H. Environmental Responsiveness of Microgel Particles and Particle-Stabilized Emulsions. Macromolecules 2006, 39, 8171-8177.

(22) Brugger, B.; Richtering, W. Emulsions Stabilized by Stimuli-Sensitive Poly(NIsopropylacrylamide)-Co-Methacrylic Acid Polymers: Microgels versus Low Molecular Weight Polymers. Langmuir 2008, 24, 7769-7777.

(23) Wiese, S.; Tsvetkova, Y.; Daleiden, N. J. E.; Spieß, A. C.; Richtering, W. Microgel Stabilized Emulsions: Breaking on Demand. Colloids Surf., A 2016, 495, 193-199.

(24) Monteux, C.; Marlière, C.; Paris, P.; Pantoustier, N.; Sanson, N.; Perrin, P. Poly(NIsopropylacrylamide) Microgels at the Oil-Water Interface: Interfacial Properties as a Function of Temperature. Langmuir 2010, 26, 13839-13846.

(25) Brugger, B.; Rosen, B. A.; Richtering, W. Microgels as Stimuli-Responsive Stabilizers for Emulsions. Langmuir 2008, 24, 12202-12208.

(26) Song, Q.; Gao, Y.; Xu, J.-F.; Qin, B.; Serpe, M. J.; Zhang, X. Supramolecular Microgels Fabricated from Supramonomers. ACS Macro Lett. 2016, 5, 1084-1088.

(27) Schmitz, D.; Pich, A. Responsive Microgels with Supramolecular Crosslinks: Synthesis and Triggered Degradation in Aqueous Medium. Polymer Chemistry 2016, 7 , $5687-5697$.

(28) Bentz, K. C.; Cohen, S. M. Supramolecular Metallopolymers: From Linear Materials to Infinite Networks. Angew. Chem. Int. Ed. 2018, 57, 14992-15001.

(29) Li, H.; Mergel, O.; Jain, P.; Li, X.; Peng, H.; Rahimi, K.; Singh, S.; Plamper, F. A.; Pich, A. Electroactive and Degradable Supramolecular Microgels. Soft Matter 2019, 15, 8589-8602.

(30) Jung, S.-H.; Schneider, S.; Plamper, F.; Pich, A. Responsive Supramolecular Microgels with Redox-Triggered Cleavable Crosslinks. Macromolecules 2020, 53, 1043-1053.

(31) Martin, R. B.; Lissfelt, J. A. The 2,2',2"'-Tripyridine System. J. Am. Chem. Soc. 1956, 78, 938-940.

(32) Constable, E. C. The Coordination Chemistry of Higher Olygopyridines 2,2'-6',2'" Terpyridine and Higher Oligopyridines. Adv. Inorg. Chem. Radiochem. 1986, 30, 69-121.

(33) Hogg, R.; Wilkins, R. G. Exchange Studies of Certain Chelate Compounds of the Transitional Metals Part VI11. 2,2 2 -Terpyridine Complexes. In Journal of The Chemical Society 1962, 341-350.

(34) Holyer, R. H.; Hubbard, C. D.; Kettle, S. F. A.; Wilkins, R. G. The Kinetics of Replacement Reactions of Complexes of the Transition Metals with 2,2',2"-Terpyridine. Inorg. Chem. 1966, 5, 622-625. 
(35) Pelton, R. H.; Chibante, P. Preparation of Aqueous Latices with NIsopropylacrylamide. Colloids Surf. 1986, 20, 247-256.

(36) Wu, X.; Pelton, R. H.; Hamielec, A. E.; Woods, D. R.; McPhee, W. The Kinetics of Poly(N-Isopropylacrylamide) Microgel Latex Formation. Colloid Polym. Sci. 1994, 272, $467-$ 477.

(37) Kano, M.; Kokufuta, E. On the Temperature-Responsive Polymers and Gels Based on N-Propylacrylamides and N-Propylmethacrylamides. Langmuir 2009, 25, 8649-8655.

(38) Kubota, K.; Hamano, K.; Kuwahara, N.; Fujishige, S.; Ando, I. Characterization of Poly(N-Isopropylmethacrylamide) in Water. Polymer Journal 1990, 22, 1051-1057.

(39) Schmatloch, S.; Schubert, U. S. Engineering with Metallo-Supramolecular Polymers: Linear Coordination Polymers and Networks. Macromol. Symp. 2003, 199, 483-497.

(40) Schubert, U. S.; Winter, A.; Newkome, G. R. Terpyridine-Based Materials; WileyVCH: Weinheim, Germany, 2011.

(41) Gohy, J.-F.; Lohmeijer, B. G. G.; Varshney, S. K.; Décamps, B.; Leroy, E.; Boileau, S.; Schubert, U. S. Stimuli-Responsive Aqueous Micelles from an ABC Metallo-Supramolecular Triblock Copolymer. Macromolecules 2002, 35, 9748-9755.

(42) Chiper, M.; Fournier, D.; Hoogenboom, R.; Schubert, U. S. Thermosensitive and Switchable Terpyridine-Functionalized Metallo-Supramolecular Poly(NIsopropylacrylamide). Macromol. Rapid Commun. 2008, 29, 1640-1647.

(43) Lohmeijer, B. G. G.; Schubert, U. S. Water-Soluble Building Blocks for TerpyridineContaining Supramolecular Polymers: Synthesis, Complexation, and PH Stability Studies of Poly(Ethylene Oxide) Moieties. Macromol. Chem. Phys. 2003, 204, 1072-1078.

(44) Collin, J. P.; Guillerez, S.; Sauvage, J. P.; Barigelletti, F.; De Cola, L.; Flamigni, L.; Balzani, V. Photoinduced Processes in Dyads and Triads Containing a Ruthenium(II)Bis(Terpyridine) Photosensitizer Covalently Linked to Electron Donor and Acceptor Groups. Inorg. Chem. 1991, 30, 4230-4238.

(45) Es Sayed, J.; Lorthioir, C.; Banet, P.; Perrin, P.; Sanson, N. Reversible Assembly of Microgels by Metallo-Supramolecular Chemistry. Angew. Chem. Int. Ed. 2020, 59, 70427048.

(46) Bellér, G.; Lente, G.; Fábián, I. Kinetics and Mechanism of the Autocatalytic Oxidation of Bis(Terpyridine)Iron(II) by Peroxomonosulfate Ion (Oxone) in Acidic Medium. Inorg. Chem. 2017, 56, 8270-8277. 
(47) Satyanarayana, T.; Anipindi, N. R. Kinetics and Mechanism of Cobalt(III) Oxidation of $\operatorname{Bis}\left(2,2^{\prime}, 6^{\prime}, 2^{\prime \prime}\right.$-Terpyridine) Iron(II) in Sulfuric Acid Solutions. React. Kinet. Catal. Lett. 1992, 47, 333-341.

(48) Chen, Y.-W. D.; Santhanam, K. S. V. Solution Redox Couples for Electrochemical Energy Storage. J. Electrochem. Soc. 1981, 128, 1460-1467. 